\title{
The Power of Sample Return Missions - Stardust and Hayabusa
}

\author{
Scott A. Sandford ${ }^{1}$ \\ ${ }^{1}$ Astrophysics Branch, NASA-Ames Research Center, \\ Mail Stop 245-6, Moffett Field, CA 94035 USA \\ email: Scott.A.Sandford@nasa.gov
}

\begin{abstract}
Sample return missions offer opportunities to learn things about other objects in our Solar System (and beyond) that cannot be determined by observations using in situ spacecraft. This is largely because the returned samples can be studied in terrestrial laboratories where the analyses are not limited by the constraints - power, mass, time, precision, etc. - imposed by normal spacecraft operations. In addition, the returned samples serve as a scientific resource that is available far into the future; the study of the samples can continue long after the original spacecraft mission is finished. This means the samples can be continually revisited as both our scientific understanding and analytical techniques improve with time.

These advantages come with some additional difficulties, however. In particular, sample return missions must deal with the additional difficulties of proximity operations near the objects they are to sample, and they must be capable of successfully making a round trip between the Earth and the sampled object. Such missions therefore need to take special precautions against unique hazards and be designed to successfully complete relatively extended mission durations.

Despite these difficulties, several recent missions have managed to successfully complete sample returns from a number of Solar System objects. These include the Stardust mission (samples from Comet 81P/Wild 2), the Hayabusa mission (samples from asteroid 25143 Itokawa), and the Genesis mission (samples of solar wind). This paper will review the advantages and difficulties of sample return missions in general and will summarize some key findings of the recent Stardust and Hayabusa missions.
\end{abstract}

Keywords. Stardust Mission, Hayabusa Mission, comets: general, comets: individual (Wild 2), asteroids, sample return

\section{Introduction}

Spacecraft are now 'routinely' used to study a host of astrophysical objects within our Solar System and beyond. The majority of these missions are designed to study their subjects remotely (space telescopes) or in situ (spacecraft sent to various destinations within the Solar System), and their use has revolutionized our understanding of space. These missions do, however, suffer from a number of limitations, particularly when it comes to determination of the detailed compositional nature of the objects studied. Sample return missions offer a means of augmenting the study of the composition of extraterrestrial objects in unprecedented detail.

The planetary sciences have long benefited from the availability of free sample returns that arrive at the Earth in the form of meteorites and cosmic dust and these materials have provided the context of much of what we know about the formation and evolution of our Solar System. However, these samples generally arrive at the Earth as 'orphans' whose original parent bodies are unknown. Under these conditions we lack the context needed to fully take advantage of the samples. Sample return missions can provide the 
material for study along with contextual information gathered from the known parent body.

Sample return began with samples returned from the Moon (Heiken et al. 1991) and these samples have amply demonstrated the advantages of sample return. These samples have now been available for over 40 years and continue to be the focus of active research. Subsequent to the lunar sample returns there was little in the way of sample return from space for many years, and what sample return there was did little to provide the parent body context of a focused sample return mission. For example, samples of extraterrestrial materials have been returned from space on exposed impact surfaces (i.e. Zolensky \& Kinard 1993), but these samples were similar to collected meteorites and stratospheric dust in the sense that the samples' parent bodies were unknown.

However, in recent years there have been a handful of missions that have flown and successfully returned to Earth with samples of targeted objects. These include the Genesis mission, which returned samples of solar wind, the Stardust mission, which returned dust samples from the coma of Comet $81 \mathrm{P} /$ Wild 2, and the Hayabusa mission, which retuned dust particles from the surface of asteroid 25143 Itokawa. All three of these missions have demonstrated the scientific value of sample return missions. In the discussions that follow, a general review of the advantages and difficulties associated with sample return missions will be presented. In addition, a number of specific examples of how sample return missions have proven their worth will be presented. These examples will focus on sample return from small, primitive Solar System objects, i.e. on the Stardust and Hayabusa missions. For further information about the Genesis mission, the reader is invited to see Burnett et al. (2003). Finally, the discussion will provide some summary comments concerning important lessons learned from these missions that are specific to sample return before ending with a brief look to what is on the horizon for future sample return missions for small Solar System bodies.

\section{Advantages of Sample Return}

There are numerous advantages to sample return for the study and understanding of our Solar System, particularly when it comes to the determination of compositions. Having actual extraterrestrial samples on hand in terrestrial laboratories allows for the use of state-of-the-art analytical techniques and equipment, providing for the ultimate in current precision, sensitivity, resolution, and reliability. In contrast, spacecraft instruments making in situ measurements are, of necessity, not state-of-the-art since they fly instrument designs that must be locked into place well before launch and frequently have extended flights to their targets. In fact, sample return missions have the advantage that they are a resource for both current and future studies. As laboratory analytical techniques improve, samples can always be reexamined to take advantage of these improvements.

Sample return missions also avoid limitations associated with cost, power, mass, and reliability that are imposed on spacecraft instruments. Spacecraft are limited on what they can carry and this constrains the measurements they can make. In contrast, samples returned to the Earth's surface can be studied using any analytical technique one cares to bring to bear, vastly increasing the information that can be gleaned from the parent body. It is interesting to note that some of the terrestrial analytical devices used to study Stardust and Hayabusa samples are not only more massive than the spacecraft that returned them, they are more massive than the launch pads from which they left the Earth's surface! 
The study of returned samples also enjoys the benefit that the samples can be studied by a broad international community that can use many different analytical techniques in an iterative and fully adaptive fashion. The work of these scientists need not be limited by instrument designs or ideas current at the time of the launch of the original spacecraft. Also, since the samples reside in terrestrial laboratories and can be made available to multiple researchers, analyses can be made using multiple, fully calibrated techniques and instruments, and verified by multiple researchers. Finally, because the actual analyses are done on Earth, sample return takes advantage of a tremendous resource not fully available to non-return missions, namely the expertise of the world's analytical chemists, physicists, and meteoriticists.

\section{Special Difficulties of Sample Return}

Of course, sample return missions also must deal with some special difficulties that do not exist, or are less problematic, for other missions. One principal additional difficulty that must be accommodated by sample return missions is that they must make round trips. Most in situ space missions arrive at their destination, do their work, and are then more-or-less abandoned when their tasks are completed. In contrast, sample return mission must not only launch, travel to their collection site, and collect a sample, but must also return to the Earth and deliver the sample safely to the surface. These issues add complications to the spacecraft's design that are associated with the sample reentry system and can make for longer mission lifetimes that place greater demands on the reliability of spacecraft systems.

Of course, one unique need that all sample return missions will share is the availability of a suitable sample collection system. The details and associated difficulties of such collection systems will vary from mission to mission and are strongly dependent on the nature of the body from which the sample is being collected, the type of sample desired, and so on. However, all such systems will share a number of common requirements. Chief among these is the need to collect, preserve, and deliver the sample with a minimum amount of alteration or contamination. Most spacecraft missions go to some pains to ensure they launch a 'clean' spacecraft to minimize risk to spacecraft operations and data taken by onboard instrumentation. Sample return missions generally share these same concerns, but have the added need to ensure the collected samples remain as pristine as is practically possible from the time of their collection to their ultimate analysis in a terrestrial laboratory. This requires that special care be taken in the design, assembly, testing, and flight of the spacecraft to mitigate contamination of the collected samples. In reality, it is generally not possible to guarantee a perfectly contamination-free environments. Thus, it is necessary for sample return missions to also make a special effort to not only carry out contamination control processes, but to also do contamination assessment. In this manner, contamination that can be eliminated is removed, and contamination that cannot is at least identified and characterized so that it will not be confused with parent body materials during analysis after sample return. Such assessments need to be made at all stages of the mission from manufacture of the spacecraft to ultimate removal of the sample from the sample return system.

While not always the case (Genesis is an example of an exceptions), sample return missions usually require the spacecraft operate in near proximity to the parent body from which the sample is to be collected. This can greatly complicate spacecraft operations and generally results in risks that are not associated with missions that have larger 'stand-off' distances. 
Finally, sample return missions are, of course, most powerful when they are equipped with many of the same sorts of in situ analytical instruments associated with one-way, non-sample return missions. Such instrumentation (imagers, spectrometer, etc.) benefit sample return missions in both practical and scientific ways. Scientifically, such measurements can greatly increase the value of the returned samples by providing context from their parent body, as well as yielding results in their own right. On a more practical note, such instrumentation can also provide a means of establishing what are the best possible sampling sites, how representative these sampling sites are of the entire body, and so on. While the additional instruments add complexity to the overall spacecraft, they generally do not raise concerns beyond those normal for non-return missions (with the exception that one needs to ensure that these instruments will not be a source of sample contamination and will work properly in the near-body sampling environment).

\section{The Stardust Comet Sample Return Mission}

\subsection{Overview of the Stardust Mission}

Stardust, a NASA Discovery class mission, was the first spacecraft in history to return solid samples from an astronomical body beyond the Earth-Moon system (Brownlee et al. 2003; Brownlee et al. 2004; Brownlee et al. 2006). The mission retrieved samples from Comet $81 \mathrm{P} /$ Wild 2 , an $\sim 4.5 \mathrm{~km}$-diameter body that was presumably formed and spent most of the past 4.5 or so billion years in the Kuiper Belt. This comet was somehow perturbed from its original orbit and on 10 September 1974 it had a close encounter with Jupiter that placed it in its current orbit, which lies between Mars and Jupiter. In its current orbit, the comet has an expected dynamical lifetime of $\sim 10^{4}$ years before it hits a larger object or is ejected from the Solar System (Levison \& Duncan 1997). Comet $81 \mathrm{P} /$ Wild 2 now approaches the Sun close enough that solar heating causes classic cometary activity near perihelion.

During the Stardust flyby on 2 January 2004, the comet was active, and images showed the presence of at least 20 dust jets coming from the nucleus (Brownlee et al. 2004; Sekanina et al. 2004) (Fig. 1). Stardust approached to within $234 \mathrm{~km}$ of the surface of Wild 2, when the comet was at a solar distance of 1.86 AU. Particles were collected from the comet's coma when they impacted at the $6.12 \mathrm{~km} / \mathrm{sec}$ encounter velocity into low-density silica aerogel (Tsou et al. 2003; Brownlee et al. 2003; Brownlee et al. 2006). The collecting aerogel consisted of a porous glass comprised of nanometer-sized silica filaments with bulk density that varied from $<0.01 \mathrm{~g} / \mathrm{cm}^{3}$ at the impact surface to 0.05 $\mathrm{g} / \mathrm{cm}^{3}$ at 3 -cm depth (Tsou et al. 2003). The aerogel's composition was predominantly $\mathrm{SiO}_{2}$, but contaminants, including some C-bearing components (largely $-\mathrm{CH}_{3}$ and $-\mathrm{CH}_{2}$ groups) were also present (Sandford et al. 2010). Stardust aerogel tiles collected over a thousand 5-300 $\mu \mathrm{m}$ (and many more smaller) comet particles. Onboard impact sensors indicate that most of the collected particles were associated with just a few specific dust jets (Tuzzolino et al. 2004). Particles ejected from the comet were exposed to space for only a few hours before collection, but solar heating was probably sufficient to vaporize most ices during transit from Wild 2 to the Stardust spacecraft. The collected materials were successfully delivered to Earth's surface by the Stardust Sample Return Capsule on 15 January 2006 (Brownlee et al. 2006), which was subsequently opened in a specially designed cleanroom at the NASA Curatorial Facility at Johnson Space Center.

Particle impacts into the aerogel produced a variety of differently shaped tracks. Non-fragmenting particles produced carrot-shaped tracks with length/diameter ratios of $>25$. However, many tracks show bulbous upper regions and sometimes multiple 'roots' 


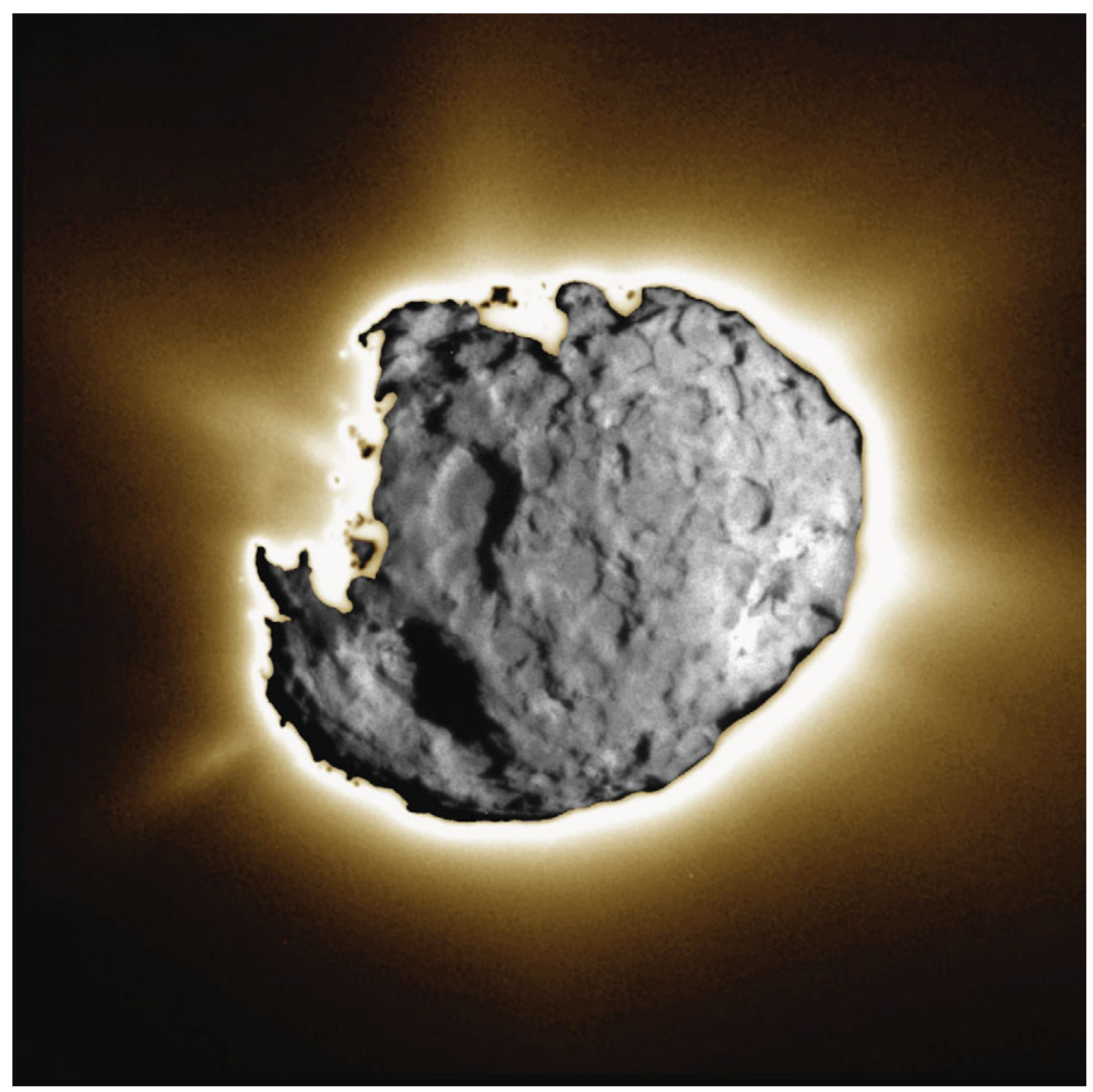

Figure 1. A composite of long and short exposure images of Comet Wild 2 showing both the complex nature of the surface of the surface of the nucleus and the many jets of dust and gas leaving its surface (see Brownlee et al. 2006).

(Fig. 2). These tracks were produced by weakly bound aggregate particles that broke apart on impact with the aerogel (Brownlee et al. 2006; Hörz et al. 2006). Typically the upper portions of tracks are lined with melted aerogel containing dissolved projectile material, the mid-track regions contain less melt and more preserved projectile material and compressed aerogel, and the ends of the tracks contain largely unmelted materials (terminal particles). The deepest penetrating particles are generally solid mineral grains or aggregates comprised of micrometer-size or larger grains. Particle impacts were also collected on the aluminum foils and frames that held the aerogel tiles in place in the collector grid. Impacts on these produced bowl-shaped craters lined with melted, and in some cases unmelted, projectile residues (Hörz et al. 2006).

For a period of six months afterwards a small fraction of these returned samples were examined by a large Preliminary Examination Team (PET) that consisted of over 175 scientists from around the world. PET members studied the samples using a host of different analytical techniques and their results have been reported in papers in special issues of Science (Brownlee et al. 2006; Flynn et al. 2006; Keller et al. 2006; McKeegan et al. 2006; Sandford et al. 2006; Zolensky et al. 2006) and Meteoritics and Planetary Science (see MAPS, vol 43, No. 1/2 2008). At the conclusion of the preliminary examination period the samples were formally turned over to the NASA Curatorial Facility to 


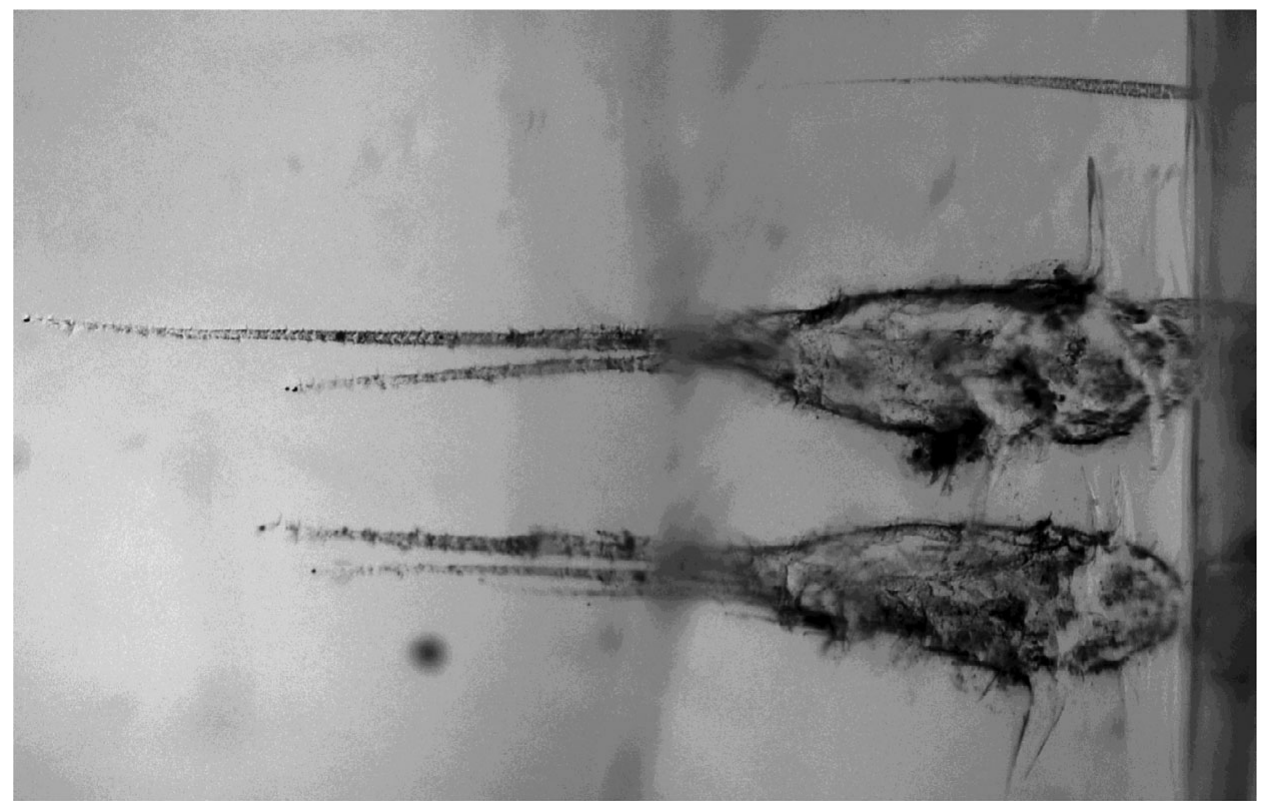

Figure 2. Many Wild 2 particles that impacted the Stardust aerogel collector were composites of many smaller grains. These particles broke up upon impact with the aerogel surface resulting in complex track forms with cometary materials deposited all along their lengths. In this image the surface of the aerogel collector is to the right and particles entered the aerogel moving from right to left. A much simpler, narrower 'carrot' track due to the impact of a single mineral grain can be seen near the top of the image. Tracks lengths depend on the size and structure of the impacting particle but are typically less than $1 \mathrm{~cm}$ in length.

be made available for allocation to the world's scientists in the same manner as Apollo samples, stratospheric cosmic dust samples, and Antarctic meteorites. Numerous samples have been distributed since that time and papers resulting from study of Stardust samples now appear regularly in the peer-reviewed literature. Some of the highlights of these studies are summarized in the section that follows.

\subsection{Highlights from Studies of the Returned Comet Wild 2 Samples}

Even in low-density aerogel, the hypervelocity capture of dust particles from Wild 2 resulted in the destruction and alteration of a significant fraction of the impacting material. This requires that care be taken to identify 'pristine' cometary materials from altered cometary materials and secondary impact products - a process made more difficult by the small nature of the samples. Despite these difficulties, it has been possible to learn an impressive number of things from the returned samples. A few points of interest and importance include:

(1) Comet $81 P /$ Wild 2 is a repository of largely unprocessed protosolar nebular materials. The distribution of minerals and organics (abundance and composition) is heterogeneous both within and between particles (Flynn et al. 2006; Sandford et al. 2006; Zolensky et al. 2006; Lanzirotti et al. 2008). These materials represent a highly unequilibrated reservoir of materials, suggesting they experienced very little parent body processing after incorporation into the comet.

(2) The protosolar nebula experienced mixing that spanned essentially its entire radial extent prior to the formation of the comet. Wild 2 samples contain everything from very high temperature minerals (Zolensky et al. 2006; Zolensky et al. 2008) to highly volatile 
organics (Sandford et al. 2006; Cody et al. 2008). These various components could not have been made at the same times in the same locations. Some must have formed very near the protosun while others could never have been inside the orbit of Jupiter and survived. And yet somehow they all got together in the comet formation zone.

(3) Comet $81 P /$ Wild 2 is not simply an assemblage of circumstellar and interstellar materials. Most of the minerals in the samples are crystalline and look very 'meteoritic' in composition and isotopic composition (McKeegan et al. 2006; Zolensky et al. 2006), implying they are of protosolar origin, not presolar origin. Isotopically anomalous circumstellar grains are seen but are relatively uncommon (Stadermann et al. 2008). Interstellar materials are more common but still represent a minor fraction of the total material (see item 5 below; McKeegan et al. 2006).

(4) Organics are present and show a large and unprecedented compositional diversity. The population of organics found in the samples are distinct from those seen in primitive meteorites and IDPs, but some show similarities to both (Sandford et al. 2006; Cody et al. 2008; Matrajt et al. 2008; Rotundi et al. 2008; Clemett et al. 2010). The organics are very rich in both oxygen and nitrogen and contain a diverse set of both aromatic and non-aromatic compounds. New materials not seen before in other extraterrestrial materials include a volatile, largely non-aromatic population that has a relatively simple chemistry (Sandford et al. 2006; Cody et al. 2008). Included in the organics is the amino acid glycine (Glavin et al. 2008) whose cometary origin has been confirmed by isotopic studies (Elsila et al. 2009).

(5) Many of the organics have an interstellar/protonebular heritage. While isotopically anomalous circumstellar grains are very uncommon, organics with excesses of $\mathrm{D}$ and ${ }^{15} \mathrm{~N}$ are less so, implying these materials or their immediate precursors have a pre-solar heritage (McKeegan et al. 2006; Stadermann et al. 2008).

In summary, the samples from Comet Wild 2 show no clear affinities with any specific meteorite type, although most of its major compositional components, particularly the minerals, are similar to those found in primitive meteorites. While some 'exotic' circumstellar and interstellar materials are present, the samples are clearly dominated by protosolar nebular materials that were apparently sampled from across the majority of the protosolar disk, perhaps over an extended period. However, the highly unequilibrated nature of the samples demonstrates that, once these materials were gathered into the comet, they experienced very little parent body processing. Thus, comets (at least as represented by Comet Wild 2) would seem to be very 'primitive' in the sense that they have preserved considerable material that was largely unaltered by parent body processes, but not particularly 'primitive' in terms of containing higher proportions of presolar materials than other available meteoritic samples. It is probably worth stressing one additional point - the enormous heterogeneity seen in the Stardust samples highlights the dangers of inferring cometary compositions on the basis of measurements, remote or otherwise, of individual species. For example, the $\mathrm{D} / \mathrm{H}$ ratio measured telescopically in an individual molecular species in a cometary coma is highly unlikely to represent a true measure of the comet's D content as a whole.

Of course, one of the advantages of sample return missions is that the items listed above represent a hard lower limit to what will be learned from these samples. The samples are still being be measured in laboratories around the world and will continue to be studied for decades into the future. Further discoveries are sure to come. 


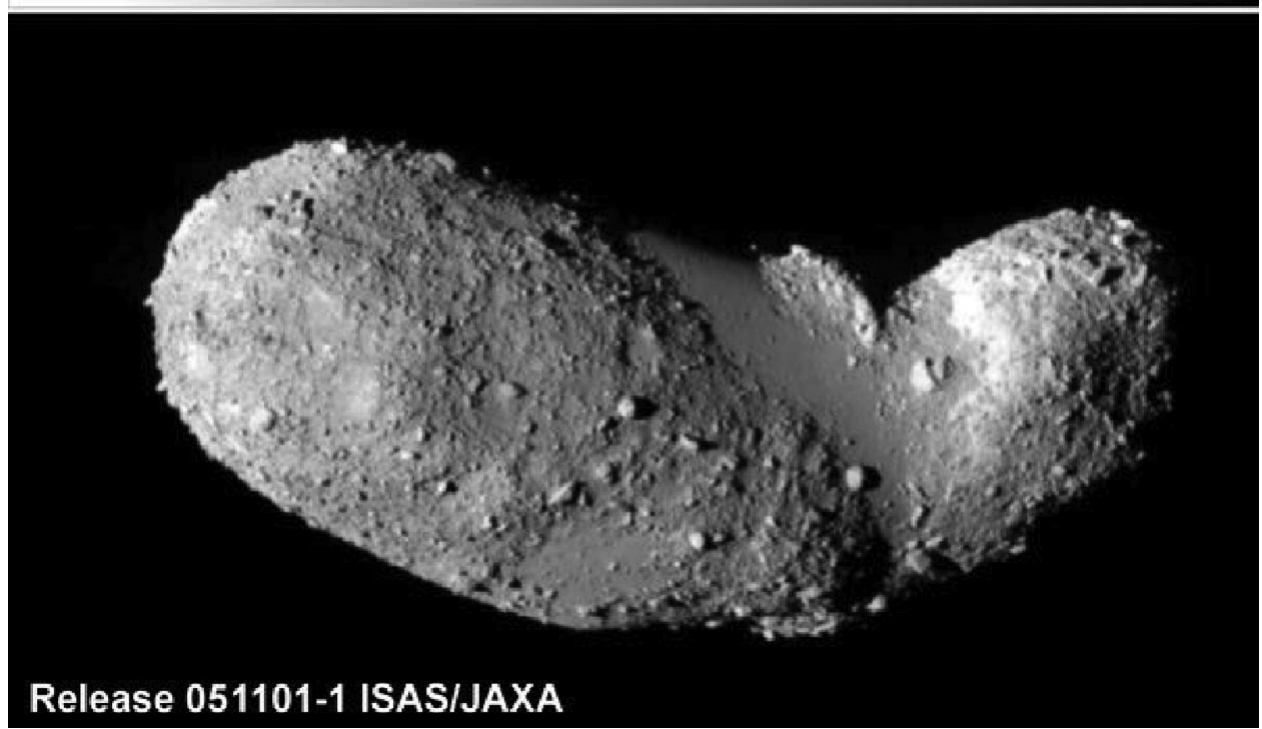

Figure 3. Asteroid Itokawa as seen by the Hayabusa spacecraft (see Yano et al. 2006). Itokawa is a small (length $\sim 540 \mathrm{~m}$ ) 'rubble pile' asteroid that shows evidence of having been collisonally disrupted in the past.

\section{The Hayabusa Asteroid Sample Return Mission}

\subsection{Overview of the Hayabusa Mission}

The Japanese Hayabusa spacecraft was launched from Earth by JAXA on an MV-5 rocket in May 2003. The spacecraft rendezvoused with its target, the small S(IV)-type asteroid 25143 Itokawa (Fig. 3), in September 2005 (Fujiwara et al. 2006), after which it carried out an extensive series of remote sensing measurements. These included measurements by an onboard near-infrared spectrometer (Abe et al. 2006) and an X-ray fluorescence detector (Okada et al. 2006). These were used to estimate the approximate mineral and chemical composition of the surface of the asteroid. The combined results of these measurements suggested that asteroid Itokawa is a 'rubble pile asteroid' consisting of materials similar to primitive LL5-6 Ordinary Chondrites. The spacecraft twice descended to the surface of Itokawa to collect samples, once on 20 November 2005 and again on 26 November 2005 in the vicinity of the MUSES-C Regio, an area on the asteroid dominated by cm-sized gravel (Yano et al. 2006). Although the spacecraft's sampling system failed to operate properly during both descents, the inlet sampling horn of this system undoubtedly impacted with the surface of the asteroid both times (Yano et al. 2006). The escape velocity of the asteroid is sufficiently small (on the order of $\sim 0.2 \mathrm{~m} / \mathrm{sec}$ ) that these touchdown impacts were sufficient to mobilize small dust grains from the asteroid's surface to the spacecraft's sample container.

Despite a number of difficulties with the spacecraft, it was ultimately able to return to Earth and successfully deliver its Sample Return Capsule (SRC) to Australia in 2010 (Abe et al. 2011). Subsequent opening of the sample canister in a specially prepared cleanroom in Sagamihara, Japan demonstrated that, while the sampling system had not recovered the full amount of material desired, it did contain thousands of particles in the 1-200 micron diameter range. Analysis of these grains has since demonstrated that these particles contain a mixture of contaminants associated with the spacecraft and dust grains from the asteroid's surface, with at least 1500 of the dust particles being asteroidal 
(Nakamura et al. 2011; Tsuchiyama et al. 2011). These samples constitute the second extraterrestrial regolith to have been sampled, the only other being the Moon, which was sampled by collections made by the Apollo and Luna missions (Heiken et al. 1991).

\subsection{Highlights from Studies of the Returned Itokawa Samples}

The Itokawa samples returned by Hayabusa have only been available for study for a relatively short time, and as a result our understanding of them is still far from complete. Nonetheless, the samples have been examined using a variety of analytical techniques and a number of important findings have already been made. A few points of interest and importance include:

(1) Itokawa materials are a match to LL Ordinary Chondrites. Particles found in the collector range from monomineralic to polymineralic and contain both poorly- and highly-equilibrated phases ( $\sim 20 \%$ and $80 \%$, respectively. The types, compositions, relative abundances and densities of minerals in the returned samples provide a good match to LL Ordinary Chondrites with the closest match being to petrographic grade LL5-6 (Nakamura et al. 2011; Tsuchiyama et al. 2011). Preliminary oxygen isotopic analyses are consistent with this identification and oxygen isotopic ratios clearly demonstrate the samples are extraterrestrial (Yurimoto et al. 2011).

(2) The returned dust particles show evidence of relatively short surface exposures. Noble gases extracted from individual grains show the presence of non-terrestrial solar wind gases whose compositions and concentrations imply relatively short surface exposures (<35 My) (Nagao et al. 2011). Additionally, approximately half of the examined particles show some evidence of space weathering at their surfaces, although the weathered layers are not as 'mature' as those seen in lunar soils, implying lower total exposures (Noguchi et al. 2011). Together, these results suggest that Itokawa has a relatively immature regolith.

The examination of samples returned by the Hayabusa spacecraft is still in its earliest stages at the time of this writing and only a small fraction of the available material has been given more than a cursory examination. It is certain that additional discovers remain to be made with these new and interesting samples.

\section{Lessons Learned from Recent Missions}

The needs and critical issues of sample return missions will vary with the nature of the samples desired and the source bodies from which they are taken. However, all sample return missions share a number of common requirements, and valuable lessons have been learned from the recent Stardust and Hayabusa missions that will be of value for future sample missions. Chief among these are issues associated with contamination control and assessment. A discussion of many of these issues in context of the Stardust mission can be found in Sandford et al. (2010). Summarized below are some lessons learned that should pertain to most sample return missions:

(1) Sample return missions need to address both contamination control, i.e., minimization of contamination, and contamination assessment, and these activities should be carried out throughout the mission from mission design to sample recovery. Contaminants should be minimized where possible, but it should also be recognized that even with contamination controls in place, there will likely still be some contamination. This is particularly problematic for collections of organic materials. Given that some contamination is likely to be inevitable, it is critical to characterize the nature of known or potential contaminants so they can be distinguished from returned samples. Ideally, 
both contamination control assessment activities should be fully integrated into a sample return mission's budget and schedule.

(2) Contamination control and assessment requires cooperative efforts be made that involve the spacecraft manufacturers, the spacecraft operators, the mission's Science Team, and whatever organization will ultimately be responsible for the distribution and longterm curation of the returned samples. Each of these groups should identify an individual or individuals responsible for organizing contamination control and assessment activities, and these individuals need to work closely together across organizational boundaries.

(3) Oddly enough, one important issue that missions need to address early is to define in a fairly rigorous way what is meant by the word 'clean' and how this definition will translate into operational activities. It is surprising how this simple word can mean very different things to different people - a spacecraft engineer may see 'clean' as an issue of eliminating surface particulates of a certain size and clean something by wiping it with a solvent soaked wipe, while an organic chemist may flinch in horror at the uniform organic residue left behind when the solvent evaporates. This is one of many areas where there are enormous benefits to clear and direct communications between mission scientists and engineers. Of course, the agreed-on definition of 'clean' may well differ from mission to mission, depending on the nature of the expected sample and how it will be analyzed.

(4) During construction of the spacecraft and sampling system, it is critical to document what components/materials are used. Where possible, samples of these materials should be collected and archived for ultimate storage at the time of manufacture. These materials can then serve as controls and standards to help assess possible contamination of the returned samples by spacecraft materials.

(5) It is important to use "witness coupons" to track the introduction of contaminates during the manufacture, flight, and recovery of the spacecraft, and during the subsequent removal of the samples from the sampling system. These coupons need to be removed and examined quickly so that problems associated with unexpected or problematic contaminants can be dealt with rapidly (again, these activities should be explicitly funded and scheduled within the mission).

(6) Witness coupons need to be designed so that they can easily be divided and distributed to multiple analysts in parallel. It is often not practical to do contamination assessment measurements using multiple analytical techniques on the same coupon in serial fashion. Sample return spacecraft should also carry a significant number of relevant witness coupons. These material represents the all-important 'blank' or 'control' sample that is critical to the interpretation of many studies of the returned samples and there will be considerable demand for them. Also, since it is not always clear what materials will work best for contamination control and assessment and different materials can result in different analytical constraints, it is generally desirable to use more than one type of witness coupon.

(7) Plans must be made in advance so the organization responsible for the ultimate curation of the returned samples is prepared to store and distribute not only the returned samples, but also the associated contamination control and assessment materials (witness coupons, samples of spacecraft materials, etc.).

\section{Future Missions}

Previous programs and missions like Apollo, Genesis, Stardust, and Hayabusa have now amply demonstrated the enormous value of returning samples to Earth for study. Given the value of these samples, and the wide variety of Solar System objects that remain unsampled, it is therefore not surprising that a number of future sample return 
missions are being considered. In terms of missions to small, primitive Solar System bodies, future sample return missions to both comets and asteroids are currently being considered and, in several cases, being built.

In the case of future missions to asteroids, there are currently two missions preparing for flight, the Japanese Hayabusa 2 mission and the NASA OSIRIS-REx New Frontiers mission. Hayabusa 2 is essentially a follow-on mission to the original Hayabusa mission and is currently targeted to visit asteroid (162173) 1999 JU3. The current schedule has a proposed launch date of July 2014 with an expected arrival at the target in 2018 . The asteroid would be surveyed for one and a half years and sampled before the spacecraft departed for Earth in December 2019, with a return to Earth in December 2020.

OSIRIS-REx was selected for flight by NASA in May 2011 and is scheduled to launch in 2016. It arrives at its target, asteroid 1999 RQ36, in late 2019 Once there, the spacecraft uses a number of instruments, including several different cameras, a laser altimeter, and two spectrometers, to take in situ observations to characterize the asteroid and establish potential sampling sites. Once a final sampling site is identified and a sample is obtained, the spacecraft will leave the asteroid in early 2021 and return its sample to the Earth on 24 September 2023.

Additional asteroid sample return missions are also in the wings, including the Marco Polo mission under consideration by ESA.

There are currently no selected comet sample return missions in flight or in preparation for flight, but a number of such missions are under consideration, and a comet sample return mission is one of the key mission types identified as important in the most recent Planetary Sciences Decadal Survey. It is therefore highly likely that one or more comet sample return missions will be in the works in the not too distant future.

\section{Conclusion}

Sample return missions provide a powerful means of learning about the nature and history of non-terrestrial bodies in our Solar System. Past sample return missions have successfully returned samples of the Moon, the solar wind, Comet 81P/Wild 2, and the asteroid 25143 Itokawa. Each of these sample returns has resulted in scientific advances that could not have been made in any other way. Combined, they amply demonstrated the value of sample return missions and strongly suggest that future such missions will yield many additional new and important insights.

\section{Acknowledgements}

The author is grateful to the organizers of this meeting for being invited to present this review and is grateful for past and current support from NASA grants from the Origins of Solar System, Exobiology, Cosmochemistry, and Discovery Mission Programs, which all have played key roles in the progression of sample return science.

\section{References}

Abe, M., et al. 2006, Science, 312, 1334

Abe, M., et al. 2011, LPSC, 42, 1638

Brownlee, D. E., et al. 2003, J. Geophys. Res., 108, 8111

Brownlee, D. E., et al. 2004, Science, 304, 764

Brownlee, D. E., et al. 2006, Science, 314, 1711

Burnett, D. S., Barraclough, B. L., Bennett, R., Neugebauer, M., Oldham, L. P., Sasaki, C. N., Sevilla, D., Smith, N., Stansbery, E., Sweetnam, D., \& Wiens, R. C. 2003, Space Sci. Rev., 105(3-4), 509 
Clemett, S. J., Sandford, S. A., Nakamura-Messenger, K., Hörz, F., \& McKay, D. S. 2010, Meteoritics and Planetary Science, 45, 701

Cody, G. D., et al. 2008, Meteoritics and Planetary Science, 43, 353

Elsila, J. E., Glavin, D. P., \& Dworkin, J. P. 2009, Meteoritics and Planetary Science, 44, 1323

Flynn, G. J., et al. 2006, Science, 314, 1731

Fujiwara, A., et al. 2006, Science, 312, 1330

Glavin, D. P., Dworkin, J. P., \& , Sandford, S. A. 2008, Meteoritics and Planetary Science, 43, 399

Heiken, G. H. et al. (eds.) 1991, Lunar Source Book, (Cambridge: Cambridge University Press), p. 736

Hörz, F., et al. 2006, Science, 314, 1716

Keller, L. P., et al. 2006, Science, 314, 1728

Lanzirotti, A., Sutton, S. R., Flynn, G. J., Newville, M., \& Rao, W. 2008, Meteoritics and Planetary Science, 43, 187

Levison, H. F. \& Duncan, M. J. 1997, Icarus, 127, 13

Matrajt, G., Ito, M., Wirick, S., Messenger, S., Brownlee, D. E., Joswiak, D., Flynn, G., Sandford, S., Snead, C., \& Westphal, A. 2008, Meteoritics and Planetary Science, 43, 315

McKeegan, K. D., et al. 2006, Science, 314, 1724

Nagao, K., et al. 2011, LPSC, 42, 2119

Nakamura, T., et al. 2011, Science, in press

Noguchi, T., et al. 2011, LPSC, 42, 1596

Okada, T., et al. 2006, Science, 312, 1338

Rotundi, A., et al. 2008, Meteoritics and Planetary Science, 43, 367

Sandford, S. A., et al. 2006, Science, 314, 1720

Sandford, S. A., et al. 2010, Meteoritics and Planetary Science, 45, 406

Sekanina, Z., Brownlee, D. E., Economou, T. E., Tuzzolino, A. J., \& Green, S. F. 2004, Science, 304,769

Stadermann, F. J., Hoppe, P., Floss, C., Heck, P. R., Hörz, F., Huth, J., Kearsley, A. T., Leitner, J., Marhas, K. K., McKeegan, K. D., \& Stephan, T. 2008, Meteoritics and Planetary Science, 43, 299

Tsou, P., Brownlee, D. E., Sandford, S. A., Hörz, F., \& Zolensky, M. E. 2003, J. Geophys. Res., 108,8113

Tsuchiyama, A., et al. 2011, Science, in press

Tuzzolino, A. J., et al. 2006, Science, 304, 1776

Yano, H., et al. 2004, Science, 312, 1350

Yurimoto, H., et al. 2011, LPSC, 42, 1755

Zolensky, M. E. \& Kinard, W. H. 1993, Adv. Space Res., 13, (8)75

Zolensky, M. E., et al. 2006, Science, 314, 1735

Zolensky, M. E., et al. 2008, Meteoritics and Planetary Science, 43, 261

\section{Discussion}

CHIAR: Can you elaborate on the size range of individual grains as well as the size(s) of the aggregates collected by the Stardust mission?

SANDFORD: Typical particles that hit the Stardust collector were in the 5-50 micron diameter range. Some of these particles were single mineral grains, but many were aggregate particles that consisted of micron and sub-micron grains. The Dust Flux Monitor detected impacts of particles up to nearly $1 \mathrm{~cm}$ in size, but these were rare and none struck the aerogel collector itself so little is known about their structure. Smaller grains $(<5$ micron diameter) also struck the collector but these are difficult to count because they create small, short tracks in the aerogel and are harder to find.

CuYlle: You mentioned the detection of amino acids in Stardust samples. Is anything known about their chirality? 
SANDFORD: Unfortunately, the only amino acid that has been identified in Stardust samples so far is glycine, which is not a chiral molecule, so nothing can currently be said about the chirality of cometary amino acids.

DE VRIES: Can you say anything about the $\mathrm{Fe} / \mathrm{Mg}$ ratios of the olivines returned by the Hayabusa mission?

SANDFORD: The suite of minerals seen in the returned Hayabusa samples, their relative abundances, and their chemical compositions are all good matches to what is seen in LL Ordinary Chondrites. Olivines, which make up approximately a third of the samples examined so far, typically show $[\mathrm{Fe} /(\mathrm{Fe}+\mathrm{Mg})]$ values that fall in the $0.25-0.35$ range, which is also consistent with LL Ordinary Chondrites. 